microvascular and macrovascular complications from diabetes), they are determined by many factors other than medical care. The problem of adjusting for case mix is one reason I do not generally favour the use of outcomes as comparative measures of quality.

Most of the measures reported by the authors, however, are process measures, are almost or entirely under the control of the doctor or practice, and are much less sensitive to the need to adjust for differences in case mix. For example, only a scale and a tape measure are needed to record body mass index, but it was done significantly less often in women, poor people, and people from ethnic minority groups. This was also the case for flu vaccination. It is hard to explain these findings other than by poorer quality of care.

So, what is to be done? I think that these data mostly show that women, poor people, and people from ethnic minority groups get poorer quality of care than do white men living in leafy areas. Some evidence shows that in the United Kingdom, financial incentives, such as those in the new general practitioner contract, will help reduce (although not eliminate) these disparities. ${ }^{5}$ I do, however, favour attempts to tweak the existing general practitioner contract to recognise that achieving the outcome targets will vary depending on where the practice is located. This could be done by weighting the payments for outcome indicators by some measure of deprivation. But this attempted tweak will come at a price: it will be more complex, for sure; it will precipitate arguments over who is deprived and how much extra the payment should be; and it may have unexpected consequences. Still, I believe the search for policy that best promotes quality, efficiency, and equity in health care is a dynamic one. The key here is both to perform adequate evaluations of the new general practitioner contract and to have policy makers who are willing to make changes when evidence accumulates that the policy is either not achieving its intended effect or producing unintended, and unwanted, side effects.

Competing interests: None declared.
1 Hippisley-Cox J, O'Hanlon S, Coupland C. Association of deprivation, ethnicity, and sex with quality indicators for diabetes: population based ethnicity, and sex with quality indicators for dis ates: population based survey in 530 Fiscella K, Franks P, Gold MR, Clancy CM. Inequality in quality: addressing socioeconomic, racial, and ethnic disparities in health care. JAMA 2000;283:2579-84

3 Epstein AM, Ayanian JZ. Racial disparities in medical care. $N$ Engl J Med 2001;344:1471-3.

4 Hippisley-Cox J, Pringle M, Crown N, Meal A, Wynn A. Sex inequalities in ischeamic heart disease in general practice: cross sectional survey. $B M I$ 2001;322:832

5 Baker D, Middleton M. Does primary care reduce health inequalities? National primary care research and development centre executive summary No 29. npcrdc.man.ac.uk/publications/29.pdf (accessed 23 Aug mary

\section{Corrections and clarifications}

Hepatitis B infections

Through editorial and production errors, we completely mangled a measurement in this editorial by Nicholas J Beeching (6 November, 1059-60). In the second sentence of the third paragraph from the end (about anti-HBs antibody seroconversion) the correct measurement in parentheses should have read, "anti-HBs antibody concentration > $10 \mathrm{IU} / 1\left[\right.$ not $\left.1 \times 10^{4} \mathrm{IU} / \mathrm{l}\right]$."

ABC of sexual health: Gender related disorders Readers may have had difficulty contacting a support group mentioned in this $\mathrm{ABC}$ article by Kevan Wylie (11 September, pp 615-7). This is because in the "web extra" information (on bmj.com) about support networks for patients undergoing gender transition we failed to double the "e" in the URL for the Mermaids support group; it should be www.mermaids.freeuk.com.

Minerva

During editing we inadvertently switched the affiliations of the second and third authors of the picture item about milker's nodule in the Minerva of 13 November ( $p$ 1194). We correctly stated that both K F To and Antonio Chuh work at the Chinese University of Hong Kong, Prince of Wales Hospital, Hong Kong, China, but K F To is professor in the department of anatomical and cellular pathology and Antonio Chuh is part time assistant professor in the department of community and family medicine (not the other way round).

\title{
bmjlearning.com
}

\section{Multimedia learning}

"Early multimedia learning looked like a car that had been cobbled together from different scrap yards with components of different sizes, colours, models, and ages. It was a mongrel beast."'

So said Donald Clark, a leading authority in multimedia learning in Britain. He was referring to the early flood of learning websites that had loud music and equally loud colour. Videos were mixed with animation, and graphics popped up all over the screen. The designers loved it, but users were at first bemused and then distracted, and in the end they learnt little. So far at BMJ Learning, we have erred on the side of multimedia learning for a purpose-you don't need broadband to use the site. Text is still very useful-experienced readers can read at a rate of 300 words a minute, but everyone can read at their own pace. You can also search text quickly, and we can easily update it.

However, we are building more multimedia content on the site. This month we have published a module on psoriasis. About 2\% of UK adults have psoriasis, and our module outlines how to diagnose and manage patients in primary care. Traditionally, colour slides are used to support text, but here the pictures are at the core of the module. We show you pictures of patients with different types of psoriasis and then test and train your skills in diagnosing and managing the condition. Further pictures enable you to test your knowledge of some complications of psoriasis such as psoriatic arthropathy and erythrodermic psoriasis. The module also gives an update on recent advances in management such as the role of vitamin $\mathrm{D}$ analogues and tumour necrosis factor blockers.

To find out more, look at our new module on bmjlearning.com.

Kieran Walsh editorial registrar, BMJ Learning

(bmjlearning@bmjgroup.com)

1 Clark D. White paper: Media rich is not always mind rich. Epic Group. www.epic.co.uk/content/resources/white_papers/media_mix.htm (accessed 29 Sep 2004). 